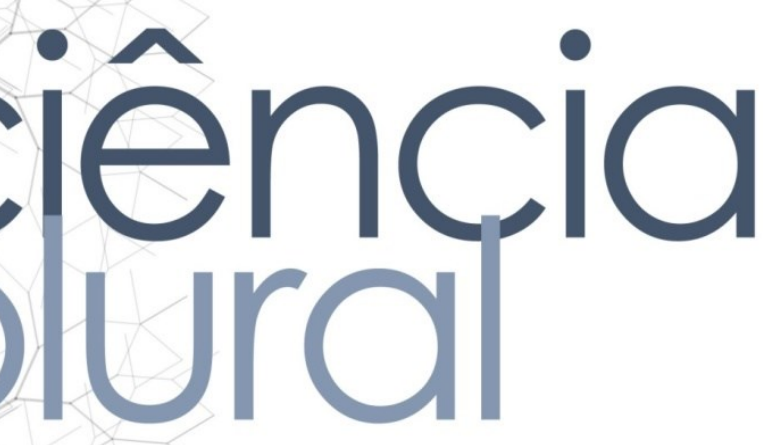

\title{
A PERSPECTIVA DO USUÁRIO NA ATENÇÃO BÁSICA SOBRE 0 ACOLHIMENTO AO IDOSO
}

\section{The user's perspective in the basic attention on the hospitality of the elderly}

Camyla Bernardo Medeiros - Universidade Federal do Rio Grande do Norte - UFRN. Mestre pelo Programa de Pós-Graduação em Saúde Coletiva. E-mail: camylabmedeiros@hotmail.com

Maria do Socorro Costa Feitosa Alves • Professora da Universidade Federal do Rio Grande do NorteUFRN. Programa de Pós-Graduação em Saúde Coletiva. E-mail: socorrocfa@hotmail.com

Luana Kelle Batista Moura • Professora do Centro Universitário UNINOVAFAPI. Teresina PI Brasil. E-mail: luana_moura19@hotmail.com

Roberta Kaliny Souza • Professora Titular da Universidade Estadual do Rio Grande do Norte UERN. Caicó RN Brasil. E-mail: robertakdesc@gmail.com

Edna Maria da Silva • Professora Titular da Universidade Federal do Rio Grande do Norte - UFRN.

E-mail: edna@reitoria.ufrn.br

Autora responsável pela correspondência:

Camyla Bernardo Medeiros•E-mail: camylabmedeiros@hotmail.com 


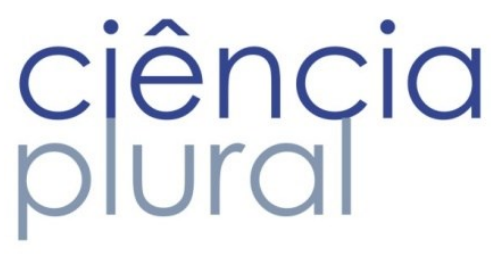

\section{RESUMO}

Introdução: 0 acolhimento, uma das diretrizes da Politica Nacional de Humanização, constitui-se em tecnologia para reorganização dos serviços, permitindo a garantia de acesso universal e humanização do atendimento. Objetivo: Este trabalho analisou a perspectiva do usuário na Atenção Básica sobre 0 acolhimento ao idoso. Metodologia: Trata-se de um estudo descritivo e analítico composto por usuários, com idade igual e superior a 60 anos, vinculados a Unidade de Saúde da Família do município de Natal/RN. A pesquisa foi aprovada com parecer $n^{0}$ 1.808.237. Para a coleta dos dados utilizou-se a entrevista semiestruturada e os dados foram analisados por meio da Análise de Conteúdo de Bardin. Resultados: Emergiram as seguires categorias: (1)Cuidado - o acolhimento foi correlacionado ao cuidado e respeito com a pessoa, visto como uma concepção ampla; (2) Acesso - verificou-se a relação direta com a espera prolongada no gerenciamento de consultas e de assistência médica; Resolutividade (3) - evidenciou novamente a burocratização do sistema de saúde e a busca por serviços privados demonstrou ser a opção de escolha. Conclusão: Concluiu-se que a perspectiva sobre o acolhimento à pessoa idosa na Atenção Básica é visto com muitas fragilidades, sobressaindo as contradições na humanização das ações em saúde.

Palavras-chave: Atenção Básica à Saúde. Acolhimento. Atenção Integral ao Idoso.

\section{ABSTRACT}

Introduction: The host, one of the guidelines of the National Humanization Policy, is a technology for the reorganization of services, allowing the guarantee of universal access and humanization of care. Objective: This study analyzed the perspective of the user in the Basic Care about the reception to the elderly. Methodology: This is a descriptive and analytical study composed of users, aged 60 and over, linked to the Family Health Unit of the city of Natal / RN. The research was approved with an opinion $n^{0} 1,808,237$. For the data collection, the semi-structured interview was used and the data were analyzed through the Bardin Content Analysis. Results: Emergent follow categories: (1) Caution - the host was correlated to care and respect with the person, seen as a broad conception; (2) Access - the direct relationship with the long wait in the management of consultations and medical care was verified; Resolutivity (3) - again evidenced the bureaucratization of the health system and the search for private services proved to be the option of choice. Conclusion: It was concluded that the perspective on the reception of elderly people in Primary Care is seen with many weaknesses, highlighting the contradictions in the humanization of health actions.

Keywords: Basic Health Care. Welcoming. Elderly. 


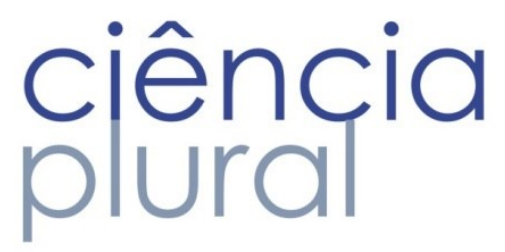

INTRODUÇÃO

A busca dos idosos pelos serviços de saúde nas Unidades Básicas de Saúde está cada vez mais presente, assim como a fragilização do acolhimento nesta demanda. Esta circunstância interfere diretamente no segmento do atendimento como um todo, resultando, muitas vezes, no aumento das hospitalizações, posto que os problemas apresentados na Estratégia de Saúde da Família- ESF apresentam pouca resolutibilidade.

O aumento da expectativa de vida da população está crescente com o passar dos anos. Dados demonstram este fenômeno, revelando que o segmento populacional que mais aumenta na população brasileira é o de idosos, com taxas de crescimento de mais de 4\% ao ano no período de 2012 a 2022. A população com 60 anos de idade ou mais passou de 14,2 milhões, em 2000, para 19,6 milhões, em 2010, devendo atingir 41,5 milhões, em 2030, e 73,5 milhões, em 2060¹.

Este fato pode ser justificado em virtude dos diversos meios de prevenção de doenças já praticados pela população, como mudança de hábitos de vida, aumento das tecnologias na área da saúde, dentre outras estratégias. Em contrapartida, aumenta também o número de pessoas acometidas por doenças crônicas ${ }^{2}$.

Em consequência, tem-se o aumento desses usuários nos serviços de saúde, especialmente na Atenção Básica, que deve ser o contato preferencial do usuário com o sistema de saúde, caracterizado por um conjunto de ações individuais e coletivas, que abrange a promoção, a prevenção e a recuperação da saúde, orientada pelos princípios da universalidade, acessibilidade e do cuidado, do vínculo, da integralidade, da responsabilização, da humanização, da equidade e da participação social, e que propõese a efetivar práticas de acolhimento e acesso a todos os níveis de atenção à saúde 3 .

O acolhimento é uma prática essencial à humanização da atenção à saúde. Com a Política Nacional de Humanização (PNH), o Ministério da Saúde reforça a valorização de atores e sujeitos que participam na produção da saúde. A operacionalização da PNH se traduz pela oferta de dispositivos (tecnologias, ferramentas e modos de operar). $O$ acolhimento se destaca pela característica de um modo de operar os processos de trabalho em saúde, de forma a dar atenção a todos que procuram os serviços de saúde, ouvindo suas necessidades e assumindo no serviço uma postura capaz de acolher, escutar e pactuar respostas mais adequadas junto aos usuários ${ }^{4}$.

Diante da importância representada pelo acolhimento e de sua repercussão para a melhoria dos serviços de saúde ao idoso, especialmente da atenção básica, porta de entrada para os serviços de saúde 


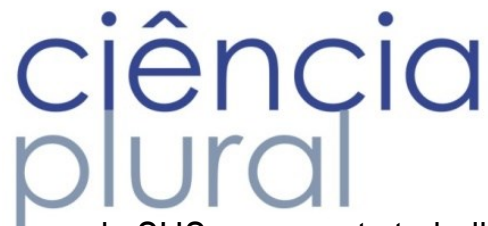

do SUS, o presente trabalho apresentou como objetivo analisar a perspectiva do usuário na Atenção Básica sobre 0 acolhimento ao idoso.

\section{INTRODUÇÃO}

\section{O envelhecimento da população}

O envelhecimento é caracterizado pelo acúmulo de danos celulares e moleculares que ocasiona perda gradual nas reservas fisiológicas e aumento do risco de adquirir doenças além de declínio da capacidade intrínseca. No Brasil, o idoso é definido pelo Estatuto do Idoso como pessoa com 60 anos de idade ou mais ${ }^{5,6}$.

A população de idosos cresce a cada ano em diversos países. Na Europa, estima-se que essa população irá atingir os 207 milhões em 2025 e 220 milhões em 2050. Além disso, dados da Organização da Nações Unidas-ONU 2002, indicam que essa proporção será de 17,5\% na Ásia e 19,3\% na Oceania no referido ano. Situação peculiar ocorre em Portugal, o qual em 2013 já apresentava 136 idosos para cada 100 jovens e estima-se que em 2053 seja de 296 idosos para cada 100 jovens $^{7}$.

Tal representação demonstra a dimensão global do envelhecimento humano. Tendo em vista 0 envelhecimento populacional que vem ocorrendo, emergem alguns desafios, tais como a questão da previdência social, saúde, assistência social, cuidado e integração social desse público".

O envelhecimento traz consigo o declínio das atividades funcionais de modo progressivo, o que torna o idoso mais suscetível a riscos diversos e a doenças. A longevidade, consequentemente implica em preocupação com os cuidados prestados no fim da vida aos idosos ${ }^{7}$. Os problemas que acometem os idosos podem resultar em aumento do número de internações hospitalares, as quais são mais frequentes e duradouras e, sendo assim, o envelhecimento implica em maior carga de doenças na população, mais incapacidades e consequentemente aumento do uso dos serviços de saúde ${ }^{8}$.

Nessa perspectiva, com a maior presença dessa população idosa nos serviços de saúde, é necessário que esses estejam preparados para recebê-los, embasados em princípios e diretrizes do Sistema Único de Saúde (SUS), como a integralidade; equidade; descentralização além de políticas de saúde capazes de contribuir com o envelhecimento ativo e saudável, bem como com um ambiente social e cultural mais favorável ao idoso. 


\section{Acolhimento em saúde}

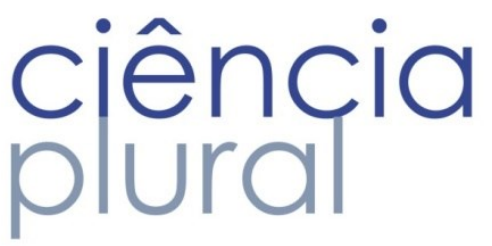

Buscando melhorar as relações existentes entre usuários e profissionais de saúde no processo de cuidado, bem como as práticas de saúde no geral, surge a Política Nacional de Humanização (PNH) elencando mudanças nas relações estabelecidas nos serviços de saúde embasando-se em princípios como protagonismo, corresponsabilidade e autonomia dos sujeitos, além de sua principal diretriz que é o acolhimento 4 .

Cuidar constitui dar atenção especial, inquietação, se dedicar a alguém ou a algo, ter responsabilidade por alguém. Tais significados são atribuídos a uma dimensão social que implica interação entre os sujeitos. Todavia, ao longo dos anos o termo tem se tornado mais voltado para atenção à saúde, esse fenômeno ocorre devido principalmente ao fato de que 0 ato de cuidar de forma mais especializada está atrelado a diagnosticar, tratar e prevenir tendo por base o conhecimento técnico e científico. Este ideal tecnicista está relacionado ao modelo biomédico, o qual foi amplamente criticado já que não considera a subjetividade do indivíduo, e por isso, a medicina atual enfatiza o respeito à subjetividade e individualidade ${ }^{9}$. Sendo assim, 0 acolhimento se constitui em instrumento fundamental para a melhoria do cuidado.

Entretanto, ainda é possivel deparar-se com uma compreensão ultrapassada, assim como a demonstrada em pesquisa feita com enfermeiros das Estratégia de Saúde da Família-ESF de Campinas/SP, onde os entrevistados relatam que 0 acolhimento se configura em uma triagem onde $\mathrm{o}$ usuário apresenta sua queixa e é realizada a conduta sem ênfase na escuta. Sendo assim, foi referido um acolhimento pontual voltado para demanda espontânea ${ }^{10}$.

Como se observa na literatura, o acolhimento deve ser implementado com a família/comunidade atendida nos serviços. Além disso, é necessário que os profissionais estejam preparados para lidar com questões relacionadas ao envelhecimento; eliminar a fragmentação do processo de trabalho integrando a atenção básica ao sistema de referência; facilitar o acesso do idoso a outros níveis de atenção e investir na qualificação dos profissionais ${ }^{10}$.

\section{METODOLOGIA}

Trata-se de um estudo descritivo/analítico com abordagem qualitativa, autorizado pelo Comitê de Ética em Pesquisa do Hospital Universitário Onofre Lopes (CEP-HUOL) sob o parecer de $n^{0} 1.808 .237$

O estudo foi realizado na Unidade básica de Saúde de Bom Pastor, município de Natal, Rio Grande do Norte, composta por quatro (04) Equipes de Saúde da Família. A escolha fundamentou-se por maioria dos moradores do bairro ser de baixa condição socioeconômica e viver no contexto de condições sanitárias 


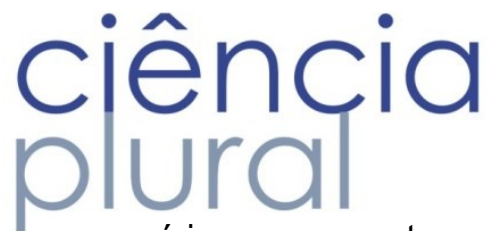

precárias, e apresentar um contingente populacional de 18.224 habitantes, sendo 8.897 homens e 9.032 mulheres. Desse total, 6,5\% são idosos os quais utilizam os serviços de saúde locais, bem como a rede implicada na Atenção Primária à Saúde e os serviços oferecidos nas demais complexidades do SUS.

Os participantes do estudo compreenderam 30 usuários, com idade a partir de 60 anos, atendidos no serviço de atenção básica, os quais integram o Programa de Hiperdia. O programa de Hiperdia contempla as seguintes patologias: Hipertensão Arterial Sistêmica (HAS) e Diabetes, as quais apresentam aspectos em comum como: etiopatogenia, fatores de risco (sedentarismo, obesidade), pode haver tratamento não medicamentoso, cronicidade, necessidade de controle rigoroso, dentre outras ${ }^{11}$.

O grupo de idosos acompanhados no Hiperdia é composto por cerca de 1360 idosos e foi utilizada uma amostra de 30 idosos, considerando o critério de saturação das respostas. Os critérios de inclusão elencados foram: ter idade igual ou superior a 60 anos; ser usuário cadastrado da ESF; estar em acompanhamento no programa hiperdia. Foram excluídos da amostra os idosos que são acompanhados apenas nas visitas domiciliárias, idosos considerados em evasão ou ser acompanhante de pacientes que estejam em atendimento na unidade.

A coleta dos dados foi realizada por meio de entrevista semiestruturada, e os dados foram coletados no período entre novembro de 2016 e fevereiro de 2017, de forma individualizada em local reservado, gravados em áudio em aparelho celular, nos dias e horários de acordo com o funcionamento e agendamento do programa Hiperdia e posteriormente transcritas para formação de banco de dados e analisadas por meio da Análise de Conteúdo ${ }^{12}$. Os dados sociodemográficos foram analisados e descritos por meio de estatística descritiva

A Análise de Conteúdo considera as significações do conteúdo, além da forma, distribuição, buscando conhecer o que está por trás das palavras, variáveis de ordem psicológica, sociológica, histórica, dentre outras, através de dedução baseada em indicadores reconstruídos a partir de uma amostra de mensagens particulares ${ }^{12}$.

As fases da Análise de Conteúdo constituem-se de: Pré-análise; Exploração do material; Tratamento dos resultados; Inferência e Interpretação ${ }^{12}$. No estudo optou-se por utilizar a categorização dos dados que teve por objetivo apreender a representação dos dados brutos visando a alcançar o núcleo de compreensão do texto.

\section{RESULTADOS E DISCUSSÃO}

Relacionado aos aspectos sócio-demográficos investigados neste estudo, dos 30 entrevistados $(63,3 \%)$ eram do gênero feminino e $(36,7 \%)$ era do gênero masculino. De modo semelhante ocorreu em estudos realizados sobre 0 acolhimento, em que a maioria dos entrevistados eram do gênero feminino 
$(85,81 \%)^{13}$. Com relação à faixa etária, foram divididas em: 60 a 70 anos: $66,6 \%$ que representou a maior parte; 71 a 80 anos (26,6\%) e acima de 81 anos (6,8\%). Ao longo dos anos, os idosos estão vivendo cada vez mais. Dados de projeção do IBGE, indicou que em 2030, a esperança de viver aos 60 anos será de 23,8 anos para ambos os sexos, atingindo até 25,2 anos em 20601.

No ponto que se refere à escolaridade pode-se notar que a maioria, $(53,3 \%)$ estudaram até 0 ensino fundamental incompleto; $(16,6 \%)$ são analfabetos; (13,3\%) estudaram o ensino fundamental completo; $(10 \%)$ o ensino médio incompleto e $(6,6 \%)$ fizeram o ensino médio completo. Na questão que trata sobre 0 trabalho/emprego observa-se variadas profissões, sendo (26,6\%) Auxiliar de Serviços Gerais (ASG); $(23,3 \%)$ agricultores; (10\%); (10\%) pedreiros; do lar (6,6\%) costureiras; $(6,6 \%)$ recepcionistas; $(3,3 \%)$ padeiro; (3,3\%) comerciante; $(3,3 \%)$ autônomo; $(3,3 \%)$ cobradora e (3,3\%) operador de máquina.

No quesito sobre a renda identificou-se que a maioria, $(80 \%)$ dos entrevistados tinha uma renda de até um salário mínimo e (20\%) recebiam de 2 a 3 salários; Nenhum dos entrevistados referiu receber mais de 3 salários mínimos. A renda familiar constatada retrata o baixo poder aquisitivo dos estudados bem como 0 baixo nível de escolaridade. $O$ baixo nível de instrução retrata o envolvimento nas ocupações apresentadas. Sobre a questão da condição de moradia $(73,3 \%)$ afirmaram morar em residência própria e (26,6\%) moram em casa alugada. Com relação ao tempo de acompanhamento na ESF, $(33,3 \%)$ são acompanhados há até 5 anos, (33,3\%) há cerca de 6 a 10 anos e (33,3\%) referiram ser acompanhados há mais de 10 anos. Essa situação pode ser entendida devido a problemas na adesão dos idosos ao longo dos anos aos programas voltados para o tratamento e controle de doenças crônicas como a hipertensão e diabetes ${ }^{14}$.

A Atenção Básica, reformulada recentemente se caracteriza como conjunto de ações individuais e coletivas de saúde que contemplam promoção, prevenção, proteção, diagnóstico, tratamento, reabilitação, redução de danos, cuidados paliativos e vigilância em saúde voltada para uma população específica e em espaço definido, por uma equipe multiprofissional. A Atenção Básica é disponibilizada de forma gratuita e integral a todas as pessoas de acordo com suas necessidades, bem como com os condicionantes de saúde ${ }^{15}$.

$\mathrm{O}$ acolhimento também definido como cuidado, se configura como um comportamento frente ao outro, caracteriza-se como uma postura de trabalho, preocupação e envolvimento com o outro. Cuidar constitui dar atenção especial, inquietação, se dedicar a alguém ou a algo, ter responsabilidade por alguém. Tais significados são atribuídos a uma dimensão social que implica interação entre os sujeitos ${ }^{9}$. 0 acolhimento inclui relações interpessoais e almeja o conforto, além de reconhecer o usuário inserido em seu contexto e com dimensões subjetivas e objetivas ${ }^{10}$. 
A PNH coloca em pauta desafios do SUS como humanizar o cuidado e dignificar o trabalho. Uma das diretrizes da $\mathrm{PNH}$, o acolhimento se configura enquanto prática essencial à qualificação e a humanização da atenção à saúde, e a avaliação dos serviços de saúde pelos usuários tem sido um importante instrumento na avaliação da qualidade dos cuidados em saúde e favorece a humanização, ademais configura-se em alternativa de analisar a resposta da comunidade à oferta do serviço disponível ${ }^{16}$.

Por meio da análise do material, na constituição do Corpus da fala dos idosos emergiram as Categorias: Cuidado; Acesso e Resolutividade que serão apresentadas a seguir respectivamente.

$\mathrm{Na}$ categoria cuidado o discurso dos idosos sobre o acolhimento, em sua maioria, aparece como uma concepção ampla extrapolando a dimensão biológica e referiram o acolhimento como cuidado, carinho, ser bem acolhido, bom atendimento e respeito, dando origem a referida categoria. O respeito ao idoso é um tema que faz parte do Estatuto do Idoso e deve ser executado pelas instituições de modo geral, inclusive pelas instituições de saúde, obedecendo aos princípios de equidade e integralidade defendidos pelo Sistema Único de Saúde.

De modo geral, o entendimento do acolhimento enquanto o cuidado visualizado pelo idoso encontrase em tangente com o defendido pela PNH quando diz que o acolhimento busca reconhecer a necessidade do outro, a criação de relações de vínculo e confiança de modo a garantir o acesso oportuno e ampliar a efetividade das práticas ${ }^{4}$.

Estudo realizado na atenção básica sobre o acolhimento aos idosos, foi constatado que $63 \%$ destes afirmaram ser escutados quando necessitam, 23,2\% relataram ter a demanda acolhida por algum profissional em cerca de 11 e 30 minutos, 40\% deles classificaram o acolhimento como bom e 51,3\% declararam que as orientações fornecidas pelos profissionais atendem sempre a necessidade deles no momento da escuta ${ }^{17}$. Alguns dos entrevistados afirmaram ter um acolhimento favorável devido ao fato de possuírem um elo com o agente de saúde, o qual se responsabiliza por marcar as consultas e informá-los sobre 0 atendimento, como relatado nas seguintes falas:

A única pessoa que me ajuda bastante aqui é a Fátima que é agente, tudo é com ela que eu sou analfabeto, ai toda hora que chego aqui ela que resolve pra mim sabe? (Ido 28).

Como eu sou hipertensa aí a gente não vem marcar a ficha, procura a agente de saúde aí ela marca pra gente e a gente vem só pra o peso e se consultar... pra mim já é um acolhimento né? (Ido 24) 
O Programa de Agentes Comunitários de Saúde (PACS) foi regulamentado em 1997 e inspirado em experiências de prevenção através de informações sobre cuidados de saúde. Os Agentes Comunitários de Saúde (ACS) são pessoas de dentro da própria comunidade que atuam junto à população, visando formar uma ligação efetiva da comunidade com as Unidades de Saúde ${ }^{11}$.

Ao ACS cabe também, como membro da equipe de saúde, realizar o acolhimento dos usuários desenvolvendo a escuta qualificada das necessidades de saúde. 0 acompanhamento realizado pelos outros membros da equipe também vai interferir na qualidade do acolhimento.

Por outro lado, encontrou-se usuários idosos que nada sabiam sobre o acolhimento, cerca de 43,3\% dos entrevistados, evidenciando um quadro comum na saúde, pela limitação na comunicação, nos aspectos educativos em saúde, na orientação sobre a prevenção de doenças, caracterizando-se na assistência médico-centrada, a qual contradiz o que é preconizado pela $\mathrm{PNH}$, que baseia-se no acolhimento como diretriz e torna os usuários atores participantes do seu processo de saúde. Nas seguintes falas pode-se observar esse déficit no conhecimento dos usuários sobre o que é acolhimento.

Sei não, tenho nem ideia, sei o que é não (Ido 6).

Só de nome eu não sei o que quer dizer não (Ido 11).

Não, sei não (Ido 20).

A falta de acolhimento pode refletir diretamente, em muitos casos, como barreira no acesso aos serviços de saúde. De acordo com Merhy ${ }^{18}$, 0 acolhimento e 0 acesso aos serviços estão intimamente relacionados, já que através da escuta qualificada e humanizada se produz vínculo, compromisso e se efetiva o acesso aos serviços.

Uma das metas da Organização Mundial da Saúde-OMS e do Ministério da Saúde é a melhoria da formação e educação continuada de profissionais da saúde para que possam avaliar e tratar as condições que afetam os idosos fornecendo ferramentas em busca de um envelhecimento saudável ${ }^{4}$.

No que se refere a categoria acesso, os serviços de saúde devem, pelo Estatuto do Idoso, em seu Capítulo IV é definido que o SUS deve garantir esse acesso de forma universal e igualitária ${ }^{6}$.

O envelhecimento traz consigo o declínio das atividades funcionais de modo progressivo o que torna 0 idoso mais susceptível a riscos diversos e a doenças. Assim o atendimento na unidade, revela muitos desafios. Os idosos caracterizaram o acesso aos serviços e o atendimento como bom em sua maioria, todavia foram elencados vários problemas como a falta do médico, a dificuldade no agendamento de consultas, a prioridade que as vezes não é respeitada, a dificuldade na contra referência e a demora para consulta, como retratado nas seguintes falas. 
Num é muito bom não, porque são poucas fichas né, na Esperança tem mais ficha que aqui, aqui só dá 8 fichas na Esperança dá 20 (Ido 5).

Eu não acho muito bom não porque é difícil que a gente que é idoso pra vim pegar uma ficha tem que vir de 4 horas ou 5:30, arriscando a vida né? Eu acho assim, era pra marcar, a gente tem prioridade né? (Ido 18)

$O$ acesso também pode ser entendido como a distância entre a unidade e a residência do indivíduo, incluindo barreiras geográficas, meio de deslocamento, dificuldades para ser atendido como: fila, tempo de espera, tratamento recebido, prioridades em situações de risco, respostas para os problemas, bem como agendamentos ${ }^{19}$.

Corroborando com este pensamento, estudos refletem que a escuta da opinião de quem recebe a assistência é um elemento importante na avaliação dos serviços. Em seu estudo ${ }^{20}$ identificou vários aspectos que dificultam o acesso, como a espera prolongada pelo atendimento, a dificuldade em conseguir consulta, o funcionamento da UBS, a organização dos serviços que não está de acordo com as necessidades da comunidade, a não percepção do usuário como ser humano completo, a falta de acompanhamento no caso de transferência para outros serviços, pouco interesse sobre a opinião do usuário acerca de seu atendimento e pouco interesse com relação às condições de vida do usuário ${ }^{20}$.

Os serviços de saúde atualmente fragmentam a atenção ao idoso com múltiplas consultas de especialidades, vários fármacos e exames, sobrecarregando o sistema. Uma linha de cuidado ao idoso eficaz deve propor uma rede articulada, referenciada e com um sistema de informação com essa lógica visando melhorar as ações em saúde voltadas a esse público².

Portanto, o acolhimento pressupõe ampliar a capacidade da equipe em dar respostas aos problemas dos usuários reduzindo a centralidade nas consultas médicas e utilizando o potencial dos demais profissionais envolvidos no processo de saúde/doença ${ }^{21}$.

Com relação à questão de acesso a exames ou encaminhamentos para outras especialidades, a maioria dos idosos afirmou ter acesso, como retratado nas falas seguintes, porém ficou bastante evidente a dificuldade devido, mais uma vez, a falta de médico e o tempo de espera prolongado para realizar os exames, os quais optavam muitas vezes em pagar pelo procedimento.

Não, nem todos. Agora até pra fazer um exame de sangue tem que tirar uma ficha, é horrivel, os outros exames a gente faz mais particular (Ido 14). 


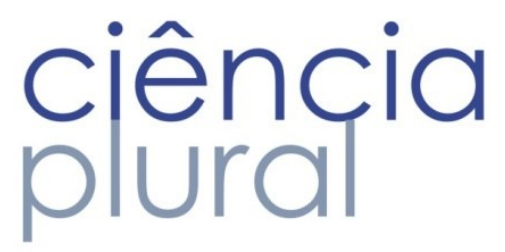

Eu tenho só pra encaminhamento, que eu preciso assim, fisioterapia, oculista, aí eles me encaminham. Exames eles passam, mas eu faço fora porque quase eu não consigo aqui porque demora muito, aí eu faço particular (Ido.12).

O estatuto do Idoso em seu capítulo IV, Art.15 trata do Direito à saúde, enfatizando a atenção integral ao idoso por intermédio do SUS garantindo acesso às ações e serviços ${ }^{6}$. A dificuldade no acesso e até indisponibilidade de exames foi um problema evidenciado o qual fere os direitos do idoso assegurados no Estatuto, sendo assim, cabe aos serviços de saúde articularem de forma mais eficaz as ações de modo que os usuários não sejam penalizados deixando de realizar as ações que necessitam.

Se faz importante que os gestores e a Estratégia Saúde da Família revejam formas de tornar o idoso uma prioridade no serviço. Na categoria acesso, os usuários apontaram a falta de assistência médica e dificuldades na realização dos exames, e isso muitas vezes, pode comprometer a resolução dos seus problemas e atendimento às suas necessidades, interferindo na resolutividade.

$\mathrm{Na}$ Categoria Resolutividade, ficou evidente a necessidade da organização dos serviços voltados para a comunidade. A resolutividade está atrelada à resolução dos problemas trazidos pelos usuários, bem como sua satisfação e dos profissionais de saúde. Essa resposta dada pode ser representada pelo alívio do sofrimento, promoção ou manutenção da saúde. De outro modo, a resolutividade pode ser investigada em dois aspectos: no serviço, com relação à capacidade de atender à própria demanda e no encaminhamento das situações que precisam de atendimento mais especializado. Esse encaminhamento inclui desde a consulta inicial, exames, tratamento e resolução final do caso em outros níveis ${ }^{22}$.

O acolhimento não se configura em um local ou espaço, e sim, implica em uma postura ética onde serão compartilhados os saberes e as dificuldades e, além disso, os profissionais deverão ter corresponsabilização com as demandas e dar resolutividade aos $\operatorname{casos}^{23}$.

A referência e contrarreferência diz respeito à organização dos serviços de saúde em redes, baseadas em fluxos e pactos de funcionamentos assegurando a atenção integral. 0 sistema de redes é estruturado em diversos níveis de complexidade e possibilita o encaminhamento entre os serviços de forma resolutiva ${ }^{24}$.

Sendo assim, para que haja um bom acolhimento por parte da equipe é necessário que o atendimento extrapole a receptividade e busque o comprometimento e a corresponsabilização com o outro. Em estudo realizado acerca da satisfação do usuário com 0 acolhimento, $79,2 \%$ dos entrevistados afirmaram ter um atendimento resolutivo, já que conseguem resolver no próprio serviço seus problemas, e somente $20,8 \%$ afirmam que o serviço deixa a desejar ${ }^{25}$. 
No presente estudo foi verificado que grande parte dos idosos realiza o pagamento de exames, entretanto a maioria dos entrevistados, cerca de $80 \%$ (24) possuem uma renda mensal de até um salário mínimo, valor esse que é utilizado para todas as necessidades do indivíduo, o que inviabiliza ainda mais o pagamento de exames em serviços privados.

A PNH refere que os usuários de saúde tem direitos garantidos por lei e os serviços de saúde devem estimular o conhecimento desses direitos e garantir que eles sejam cumpridos, com efeito a questão do acolhimento e acesso se colocam como de grande relevância ao idoso, e a população como um todo, considerando os benefícios que traz ao usuário, bem como aos serviços de saúde. Ademais, através do acolhimento com boa escuta, pode-se possibilitar o acesso aos diversos níveis de complexidade através de referência e contrarreferência, possibilitando maior resolutividade das suas demandas.

\section{CONCLUSÕES}

O presente estudo concluiu que os idosos compreendem o acolhimento como cuidado, carinho e respeito com a pessoa, trazendo questões que vão além da visão tradicional, voltada para o acolher como recepcionar. Mesmo com os avanços trazidos pelo SUS ainda é evidente o modelo de atenção traduzida pelo controle no número de consultas médicas e longa espera em filas. Diante do exposto vale ressaltar que apesar de existir essa compreensão, na prática ainda se observa um acolhimento tradicionalista. Este cenário pode ser modificado a partir do momento em que as equipes começarem a implantar a Política Nacional de Humanização em suas ações, com a capacitação dos profissionais e melhorias nas condições de trabalho dos envolvidos no processo, buscando a prática do acolhimento, a satisfação e resolução dos problemas dos usuários, além de maior discussão do tema nas universidades, viabilizando uma formação mais voltada para essa perspectiva, assim como a gestão do sistema de saúde que deve buscar articular de forma mais eficaz as ações de saúde de modo que os usuários não sejam penalizados.

Esta pesquisa reforça a importância dos novos estudos sobre acolhimento ressaltando a necessidade de reflexões, além de pesquisas que ampliem o atendimento às necessidades dos usuários idosos. Enfatizase que acesso e acolhimento se colocam como desafios ao SUS, como um processo em construção, sendo fundamental para o estudo e prática da gestão e avaliação dos serviços de saúde. 


\section{REFERÊNCIAS}

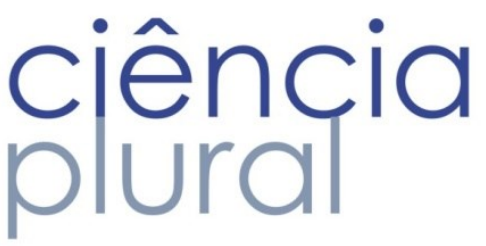

1. Instituto Brasileiro de Geografia e Estatística. (IBGE). Mudança Demográfica no Brasil no início do século XXI: Subsídios para as projeções da população. Rio de Janeiro. 2015; (3). Estudos e Análises. Informação Demográfica e Socioeconômica.

2. Veras R. Linha de Cuidado para o Idoso: detalhando o modelo. Rev Bras Geriat Gerontol. 2016; 19 (6):887-905.

3. Brasil. Ministério da Saúde. Portaria $n^{0} 2.488$, de 21 de Outubro de 2011. Política Nacional de Atenção Básica. 2011 [Acesso em: 25 de abr. 2017]. Disponivel em: $<$ http://bvsms.saude.gov.br/bvs/saudelegis/gm/2011/prt2488_21_10_2011.html>.

4. Brasil. Ministério da Saúde. Política Nacional de Humanização: PNH. Humaniza/SUS. Brasília: DF. 2013b. Ministério Da Saúde.

5. Organização Mundial de Saúde (OMS). Relatório Mundial de Envelhecimento e Saúde. Genebra: OMS; 2015.

6. Brasil. Estatuto do Idoso. Legislação sobre o Idoso: Lei n010.741 de 01 de Outubro de 2003 e legislação correlata. Brasília: 2013a; (3). Câmara dos Deputados.

7. Carvalho MS, Martins JSA. O cuidado paliativo a idosos institucionalizados: Vivência dos ajudantes de ação direta. Rev Bras Geriatr Gerontol, 2016;19 (5):745-758.

8. Veras R. Envelhecimento populacional contemporâneo: demandas, desafios e inovações. Rev Saúde Pública, 2009; 43 (3):548-54.

9. Contatore AO, Malfitano APS, Barros NF. Os Cuidados em Saúde: Ontologia, hermenêutica e teleologia. Interface, 2017; 21(62): 553-63.

10. Costa PCP, Garcia APRF, Toledo VP. Acolhimento e cuidado de Enfermagem: Um estudo fenomenológico. Texto Contexto Enferm, 2016; 25(1).

11. Brasil. Ministério da Saúde. Departamento de Atenção Básica. Área Técnica de Diabetes e Hipertensão Arterial: Protocolo. Brasília: Ministério da Saúde; 2001.

12. Bardin L. Análise de Conteúdo. $1^{\text {a }}$ ed. São Paulo; 2011.

13. Lucena LN. O olhar do Usuário acerca do acolhimento no Município de Recife. In: Reichert APS. (Orgs.). Avaliação e gestão do cuidado na saúde da família. Fortaleza: Edições UFC; 2016. 


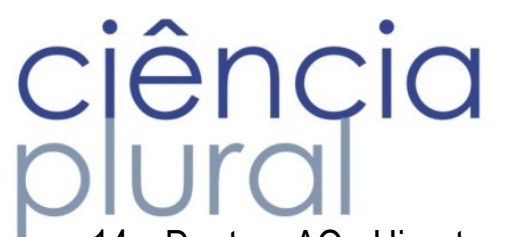

14. Dantas AO. Hipertensão Arterial no Idoso: Fatores dificultadores para a adesão ao tratamento medicamentoso. Monografia (Especialização em Atenção Básica em Saúde da Família). Belo Horizonte: Universidade Federal de Minas Gerais; 2011.

15. Brasil. Ministério da Saúde. Portaria n $n^{\circ}$ 2.436, de 21 de Setembro de 2017. Política Nacional de Atenção Básica. Brasília: 2017; Ministério da Saúde. [Acesso em: 10 de jul. de 2017]. Disponível em: $<$ <ttp://dabsistemas.saude.gov.br/portaldab/noticias.php?conteudo=_\&cod=2457>.

16. Gouveia GC, Souza WV, Luna CF, Szwarcwald CL, Souza Júnior PRB. Satisfação dos usuários com a assistência de saúde no estado de Pernambuco, Brasil, 2005. Ciênc. Saúde Coletiva [internet]. 2011; 16 (3):1849-1861.

17. Marcolino EC, Clementino FS, Almeida LAL, Nunes MLA, Miranda FAN. Acolhimento na Atenção Básica a idosos Paraibanos: Análise a partír da avaliação externa do PMAQ-AB. Anais CIEH. 2015; 2 (1).

18. Merhy EE, Magalhães Júnior HM, Rimoli J, Franco TB, Bueno WS (Orgs). O trabalho em saúde: Olhando e experienciando o SUS no cotidiano. São Paulo: Hucitec; 2003.

19. Alves SGS. Prática do acolhimento à pessoa idosa na atenção básica: Uma estratégia de Humanização. 114 f. [Dissertação] Universidade Federal do Rio Grande do Norte; 2009.

20. Lira MRS, Coutinho NPS. Avaliação do Acolhimento nas Unidades de Saúde da Família em São LuísMA. In: Reichert A PS (Orgs.). Avaliação e gestão do cuidado na saúde da família. Fortaleza: edições. UFC; 2016.

21. Ferreira MLSM, Penques RMVB, Marin MJS. Acolhimento na percepção dos enfermeiros da Atenção Primária à Saúde. Aquichan. 2014; 14 (2):216-225.

22. Turrini RNT, Lebrão ML, Galvão CCL. Resolutividade dos serviços de saúde por inquérito domiciliar: percepção do usuário. Cad. Saúde Pública. 2008; 24(3): 663-674.

23. Ribeiro $L C C$, Rocha $R L$, Ramos-Jorge $M L$. Acolhimento às crianças na atenção primária à saúde: $U m$ estudo sobre a postura dos profissionais das equipes de saúde da família. Cad. Saúde Pública. 2010; 26(12): 2316-2322.

24. Costa SM, Ferreira A, Xavier LR, Guerra PNS, Rodrigues CAQ. Referência e contrarreferência na Saúde da Família: Percepção dos profissionais de saúde. Rev. APS. 2013; 16(3): 287-293.

25. Medeiros FA, Araújo-Souza GC, Albuquerque-Barbosa AA, Costa ICC. Acolhimento em uma Unidade Básica de Saúde: A satisfação do usuário em foco. Rev. Salud. Pública. 2010; 12 (3): 402-413. 